\title{
Knockdown of $\beta$-catenin by siRNA influences proliferation, apoptosis and invasion of the colon cancer cell line SW480
}

\author{
KUI LI, ZHONG-YIN ZHOU, PAN-PAN JI and HE-SHENG LUO \\ Department of Gastroenterology, Renmin Hospital, Wuhan University, Wuhan, Hubei 430060, P.R. China
}

Received March 8, 2015; Accepted March 18, 2016

DOI: $10.3892 / 01.2016 .4481$

\begin{abstract}
The aim of the present study was to explore the effect of knocking down the expression of $\beta$-catenin by small interference (si)RNA on the activity of the Wnt/ $\beta$-catenin signaling pathway, and the proliferation, apoptosis and invasion abilities of the human colon cancer cell line SW480. For that purpose, double-stranded siRNA targeting $\beta$-catenin ( $\beta$-catenin-siRNA) was synthesized and transfected into SW480 cells. Reverse transcription-polymerase chain reaction (RT-PCR) and western blotting were used to detect the messenger (m)RNA and protein levels of $\beta$-catenin in SW480 cells. To detect cell proliferation, 3-(4,5-dimethylthiazol-2-yl)-2,5-diphenyltetrazolium bromide assay was performed, while cell apoptosis and caspase- 3 activity were detected by flow cytometry and caspase- 3 activity assay, respectively. Matrigel invasion assay was performed to detect the influence of siRNA-mediated gene silencing on the invasion and metastasis of SW480 cells in vitro. The results of RT-PCR and western blot analysis demonstrated that, compared with the blank control, negative control and liposome groups, $\beta$-catenin-siRNA transfected SW480 cells had significantly decreased mRNA and protein levels of $\beta$-catenin. In addition, following $\beta$-catenin-siRNA transfection, the proliferation of SW480 cells was significantly lower than that of the blank control, negative control and liposome groups, while the apoptosis rate increased in $\beta$-catenin-siRNA transfected cells, compared with the aforementioned groups. Invasion assay showed that, following $\beta$-catenin-siRNA transfection, the number of SW480 cells infiltrating through the Matrigel membrane was significantly lower than that of the blank control, negative control and liposome groups. Following $\beta$-catenin-siRNA transfection, the caspase-3 activity in SW480 cells was lower than that in the blank control, negative control and liposome groups. These results indicate that siRNA-mediated silencing of $\beta$-catenin
\end{abstract}

Correspondence to: Professor Zhong-Yin Zhou, Department of Gastroenterology, Renmin Hospital, Wuhan University, 238 Jiefang Road, Wuhan, Hubei 430060, P.R. China

E-mail: zhouhu0425@163.com

Key words: $\beta$-catenin gene, colorectal cancer, RNA interference, cell proliferation, apoptosis could inhibit the proliferation and invasion of SW480 cells and induce apoptosis, thus providing novel potential strategies for the clinical treatment of colon cancer, and may serve as a novel target for cancer therapy.

\section{Introduction}

The Wnt/ $\beta$-catenin signaling pathway is important in embryogenesis and tumor development, and has been recognized as one of the key signaling pathways during tumorigenesis $(1,2)$. The aberrant activation of the $\mathrm{Wnt} / \beta$-catenin signaling pathway may increase cell proliferation, which results in the generation of abnormal proteins, thereby accelerating the formation of tumors and carcinogenesis through the expression of downstream target genes (3). A previous study demonstrated that $\sim 90 \%$ of cases of colon cancer were associated with excessive activation of this pathway (4). $\beta$-catenin is the key molecule of the Wnt/ $\beta$-catenin signaling pathway (5). Thus, silencing the $\beta$-catenin gene may inhibit the development and invasion of colon cancer. However, data regarding how to influence colon cancer cells by silencing the $\beta$-catenin gene are limited. In the present study, the expression of the $\beta$-catenin gene in the Wnt/ $\beta$-catenin signaling pathway was silenced by small interference (si)RNA to study its effect on colon cancer cell proliferation, apoptosis and invasion, and to explore the clinical value of blocking $\beta$-catenin gene expression as a therapeutic molecular target.

\section{Materials and methods}

Materials. The human colon cancer cell line SW480 was purchased from the China Center for Type Culture Collection of Wuhan University (Wuhan, China). The liposomal transfection agent Lipofectamine ${ }^{\circledR} 2000$ was purchased from Invitrogen (Thermo Fisher Scientific, Inc., Waltham, MA, USA). Reverse transcription-polymerase chain reaction (RT-PCR) kit was purchased from Fermentas (Thermo Fisher Scientific, Inc.). Matrigel was purchased from BD Biosciences (Franklin Lakes, NJ, USA), while Transwell chambers were purchased from Santa Cruz Biotechnology, Inc. (Dallas, TX, USA).

Synthesis of $\beta$-catenin-siRNA. siRNA targeting the $\beta$-catenin gene was synthesized by Shanghai GenePharma Co., Ltd. (Shanghai, China), and its sequence was: Sense, 5'-CATGUG 
UTGGUAAGCUCUA-3' and anti-sense, 5'-GCAACAGTT GCAGAGAGGU-3'. Upon synthesis, Basic Local Alignment Search Tool analysis was performed in GeneBank (blast.ncbi. nlm.nih.gov/Blast.cgi) excluding other homologous genes. The negative control siRNA used in the present study was purchased from Shanghai GenePharma, Co., Ltd., and the sequence was as follows: Sense, 5'-AUGCUGATCAGUGUCGATU-3' and anti-sense, 5'-CAGAGAGCTCGUGAGAGTA-3'.

Transfection of the human colon cancer cell line SW480. SW480 cells were transfected with the Lipofectamine ${ }^{\circledR} 2000$ transfection reagent, according to the manufacturer's protocol. The human colon cancer cell line SW480 was conventionally cultured in Dulbecco's modified Eagle's medium containing $10 \%$ fetal bovine serum (FBS) (Shanghai Solarbio Bioscience $\&$ Technology Co., Ltd., Shanghai, China) at $37^{\circ} \mathrm{C}$ in a $5 \% \mathrm{CO}_{2}$ humidified incubator. Cells in the logarithmic growth phase were selected for transfection. Samples were divided into the following four groups: i) Blank control group (non-transfected cells); ii) negative control group (cells transfected with negative control-siRNA); iii) liposome group (cells transfected only with the aforementioned liposomal transfection agent); and iv) transfected group (cells transfected with $\beta$-catenin-siRNA).

RT-PCR assay for the detection of siRNA efficiency on $\beta$-catenin gene expression. Total RNA was extracted using TRIzol (Shanghai GenePharma, Co., Ltd.), and next subjected to RT reaction. RT-PCR experiments were performed using the AccessQuick RT-PCR System (Promega Corporation, Madison, WI, USA) using $1 \mu \mathrm{g}$ total RNA. The primers were purchased from Shanghai GenePharma, Co., Ltd. and the sequences were as follows: $\beta$-catenin, forward 5'-AAAGCG GCTGTTAGTCACTGG-3' and reverse 5'-GACTTGGGA GGTATCCACATCC-3'; $\beta$-actin, forward 5'-GAAATCGTG CGTGACATTA-3' and reverse 5'-ACTCATCGTACTCCT GCTTG-3'. The expected lengths of the amplified products for $\beta$-catenin and $\beta$-actin were 132 and $475 \mathrm{bp}$, respectively. The PCR was performed on a thermal cycler (Mastercycler Nexus; Eppendorf China, Ltd., Shanghai, China) under the following conditions: $94^{\circ} \mathrm{C}$ for $2 \mathrm{~min}$, followed by incubation at $94^{\circ} \mathrm{C}$ for $45 \mathrm{sec}, 55^{\circ} \mathrm{C}$ for $45 \mathrm{sec}$ and $72^{\circ} \mathrm{C}$ for $45 \mathrm{sec}$, for a total of 32 cycles, and a final step at $72^{\circ} \mathrm{C}$ for $5 \mathrm{~min}$. The results were semiquantitatively analyzed using $3 \%$ agarose gel electrophoresis (Submarine Electrophoresis Unit; Hoefer ${ }^{\mathrm{TM}}$; Thermo Fisher Scientific, Inc.) and visualized using a digital gel documentation system (GelDoc 2000 System/QuantityOne software; Bio-Rad Laboratories, Inc., Hercules, CA, USA).

Western blotting assay for the detection of siRNA efficiency on $\beta$-catenin protein expression. Four groups of SW480 cells (Blank control; negative control; liposome; transfected) were washed with phosphate-buffered saline (PBS) and lysed with lysis buffer $(150 \mathrm{mmol} / \mathrm{l} \mathrm{NaCl}, 50 \mathrm{mmol} / \mathrm{l}$ Tris, $1 \%$ sodium deoxycholate, $0.1 \%$ sodium dodecylsulfate, $1 \%$ Triton X-100, $5 \mathrm{mmol} / \mathrm{l}$ ethylene diaminetetra acetate; Shanghai Solarbio Bioscience \& Technology Co., Ltd.) for $20 \mathrm{~min}$ on ice and then centrifuged $(4,500 \mathrm{x}$ g for $10 \mathrm{~min})$. Protein levels were determined using the Pierce BCA Protein Assay $\left(\right.$ Pierce $^{\mathrm{TM}}$; Thermo Fisher Scientific, Inc.), according to the manufacturer's protocol. Next, equal aliquots of the cell lysates were run on $10 \%$ sodium dodecyl sulfate-polyacrylamide gel and then electrotransferred onto polyvinylidene fluoride membranes (Shanghai Solarbio Bioscience \& Technology Co., Ltd.), which were blocked with $5 \%$ skimmed milk powder dissolved in Tris-buffered saline with Tween ${ }^{\circledR} 20$ for $2 \mathrm{~h}$ at $4^{\circ} \mathrm{C}$. The membranes were incubated with polyclonal rabbit anti- $\beta$-catenin (catalog no. sc-7199) and mouse monoclonal anti- $\beta$-actin (catalog no. sc-47778) antibodies (dilution, 1:500; Santa Cruz Biotechnology Inc., Dallas, TX, USA) overnight at $4^{\circ} \mathrm{C}$, then with a secondary goat anti-rabbit IgG antibody (catalog no. G21079; 1:1,000; Invitrogen ${ }^{\mathrm{TM}}$; Thermo Fisher Scientific, Inc.) for $2 \mathrm{~h}$ at room temperature. Chemiluminescence (Shanghai Solarbio Bioscience \& Technology Co., Ltd.) and optical density (OD) analysis were used to detect the expression levels of $\beta$-catenin. $\beta$-actin served as an internal reference.

Effect of siRNA on cell proliferation by 3-(4,5-dimethylthiazol-2-yl)-2,5-diphenyltetrazolium bromide (MTT) assay. SW480 cells on logarithmic growth phase were trypsinized (Shanghai Solarbio Bioscience \& Technology Co., Ltd.) and seeded in 96-well plates (Shanghai Solarbio Bioscience \& Technology Co., Ltd.) at a density of $5 \times 10^{3}$ cells $/ \mathrm{ml}$. Upon 24-h incubation, cells were transfected for $24,48,72$ or $96 \mathrm{~h}$. Next, $200 \mu 1$ MTT solution was added, and incubation continued for additional $4 \mathrm{~h}$. Subsequently, the cell supernatant was discarded, and $100 \mu 1$ dimethyl sulfoxide (Shanghai Solarbio Bioscience \& Technology Co., Ltd.) was added. Plates were gently rocked at room temperature for $15 \mathrm{~min}$, and the OD at $570 \mathrm{~nm}$ was measured with a microplate reader (ELx800; BioTek, Winooski, VT, USA). A growth curve was generated by plotting the incubation time in the horizontal axis and the OD values is the vertical axis.

Flow cytometry for the detection of cell apoptosis. Apoptosis was detected using Annexin V/propidium iodide double staining (Shanghai Solarbio Bioscience \& Technology Co., Ltd.). Cells in each group were collected, digested and resuspended in PBS. Flow cytometry (BD FACSCalibur ${ }^{\mathrm{TM}}$; BD Biosciences) was used to detect cell apoptosis, according the protocol provided by the manufacturer of the kit. In the flow cytometry plots, the lower left quadrant represented cell debris, while the lower and upper right quadrant represented early and late apoptotic cells, respectively. The upper left quadrant corresponded to dead cells. The apoptotic rate for each group was calculated as the average of three independent measurements, according to the following formula: Apoptosis rate $(\%)=$ (number of apoptotic cells/number of total cells) x 100 .

Matrigel transmembrane invasion assay for the detection of cell invasiveness in siRNA-transfected SW480 cells. The Matrigel transmembrane invasion assay was performed in a Transwell plate (Corning, Inc., Corning, NY, USA). Complete medium was added to the upper chamber of Transwell plate pretreated with Matrigel (BD Biosciences), and complete medium containing $10 \%$ FBS was added to the lower chamber, following incubation for $1 \mathrm{~h}$ at $37^{\circ} \mathrm{C}$. The experimental and control groups were suspended at a cell density of $1 \times 10^{5}$ cells $/ \mathrm{ml}$, and $200 \mu \mathrm{l}$ of these suspensions were transferred to the upper 
A

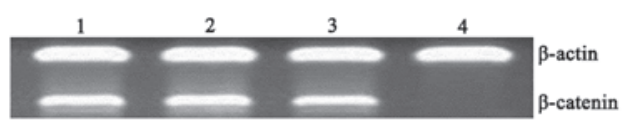

B

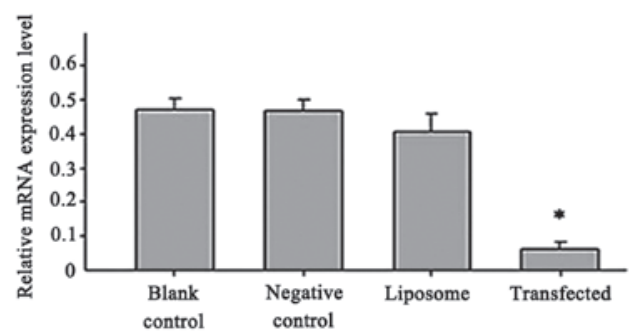

Figure 1. (A) Reverse transcription-semiquantitative polymerase chain reaction detection of $\beta$-catenin messenger RNA in the (1) blank control, (2) negative control, (3) liposome and (4) transfected groups. (B) Expression of $\beta$-catenin was significantly reduced in the $\beta$ catenin small interfering RNA experimental group compared with the blank control, negative control and liposome groups. ${ }^{*} \mathrm{P}<0.05$.

chamber, followed by incubation for $24 \mathrm{~h}$. Subsequently, cells on the filter membranes were harvested, and the number of cells was counted under a microscope (IX71; Olympus Corporation, Tokyo, Japan).

Caspase-3 activity assay to detect the influence of siRNA on the caspase-3 activity in SW480 cells. Caspase-3 activity was measured using a kit (Shanghai GenePharma Co., Ltd.) that contained caspase-3 sequence-specific peptides conjugated to a chromophore. When the substrate was cleaved by caspase-3, the chromophoric group was released, which could be monitored by measuring the OD of the sample at a wavelength of $405 \mathrm{~nm}$ on a microplate reader, thus obtaining the relative activity of caspase- 3 . The experiments were conducted according to the manufacturer's protocol.

Statistical analysis. Statistical analysis was performed using SPSS version 17.0 software (SPSS Inc., Chicago, IL, USA). Data were expressed as the mean \pm standard deviation. Student's $t$-test and one-way analysis of variance were used to compare the means across different groups. $\mathrm{P}<0.05$ was considered to indicate a statistically significant difference.

\section{Results}

$\beta$-catenin-siRNA inhibits $\beta$-catenin messenger (m)RNA expression. The results presented in Fig. 1 indicate that, following $\beta$-catenin-siRNA transfection for $48 \mathrm{~h}$, the relative mRNA expression levels of $\beta$-catenin in $\beta$-catenin-siRNA transfected SW480 cells were $0.044 \pm 0.003$, while in the blank control, negative control and liposome groups, the relative mRNA expression levels of $\beta$-catenin were $0.465 \pm 0.063,0.468 \pm 0.037$ and $0.417 \pm 0.066$, respectively. These results indicate that the expression of $\beta$-catenin was significantly reduced in the $\beta$-catenin-siRNA experimental group ( $\mathrm{P}=0.004)$, compared with the blank control, negative control and liposome groups. No significant differences were observed among the blank control, negative control and liposome groups regarding the mRNA expression levels of $\beta$-catenin $(\mathrm{P}=0.611)$. Therefore, $\beta$-catenin-siRNA effectively inhibited the expression of the $\beta$-catenin gene in SW480 cells.
A

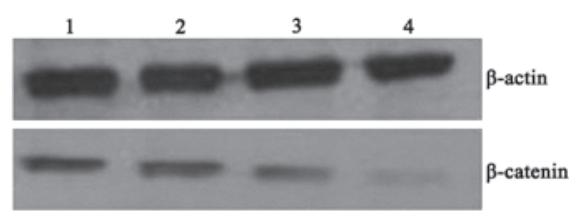

B

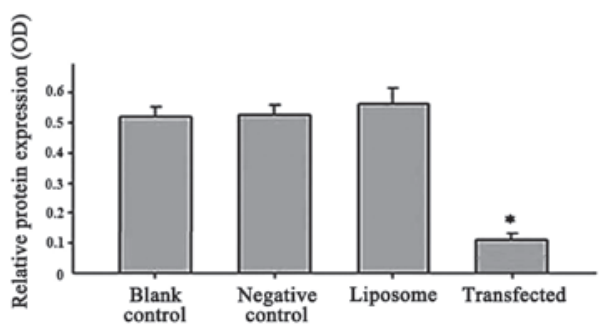

Figure 2. Small interference RNA targeting $\beta$-catenin was transfected into SW480 cells to detect the expression of $\beta$-catenin protein. (A) Western blot results of (1) blank control, (2) negative control, (3) liposome and (4) transfected groups. (B) In the transfected group, the protein expression levels of $\beta$-catenin were significantly lower compared with the blank control, negative control and liposome groups. ${ }^{*} \mathrm{P}<0.05$.

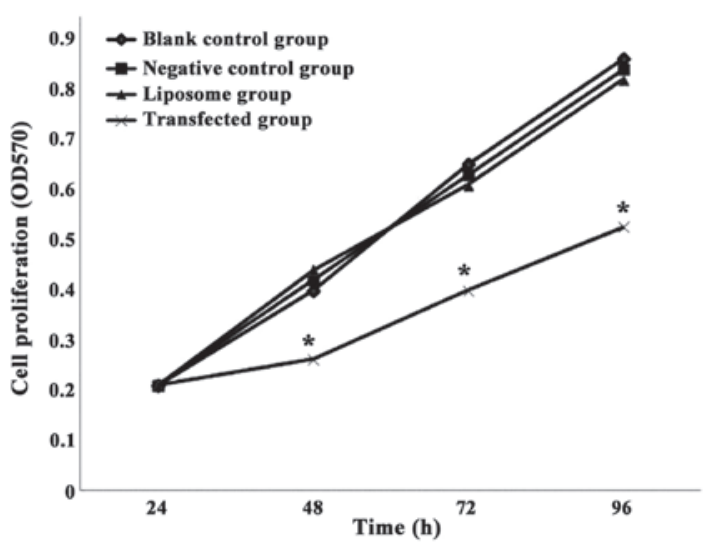

Figure 3. Inhibition of proliferation of SW480 cells was detected by 3-(4,5-dimethylthiazol-2-yl)-2,5-diphenyltetrazolium bromide assay. ${ }^{*} \mathrm{P}<0.05$ vs. blank control, negative control or liposome groups. OD, optical density.

$\beta$-catenin siRNA inhibits the protein expression of $\beta$-catenin. The results represented in Fig. 2 reveal that, following $\beta$-catenin-siRNA transfection for $48 \mathrm{~h}$, the OD value of $\beta$-catenin protein expression in SW480 cells was $0.102 \pm 0.005$, while in the blank control, negative control and liposome groups the OD values were $0.512 \pm 0.035,0.519 \pm 0.047$ and $0.566 \pm 0.036$, respectively. Thus, in the transfected group, the protein expression levels of $\beta$-catenin were significantly lower than in the other groups $(\mathrm{P}=0.014)$. No significant differences were observed among the blank control, negative control and liposome groups in terms of $\beta$-catenin protein expression $(\mathrm{P}=0.237)$. Thus, $\beta$-catenin-siRNA effectively inhibited the protein expression of $\beta$-catenin in SW480 cells.

$\beta$-catenin-siRNA inhibits the proliferation of SW480 cells. The results presented in Fig. 3 indicate that, following $\beta$-catenin-siRNA transfection for $24 \mathrm{~h}$, there was no significant difference in SW480 cell proliferation in the $\beta$-catenin-siRNA-transfected group, compared with the blank control, negative control and liposome groups. However, upon 
Table I. Influence of small interference RNA targeting $\beta$-catenin on the apoptosis rate and caspase-3 activity of SW480 cells.

\begin{tabular}{lcc}
\hline Group & $\begin{array}{c}\text { Cell apoptosis rate, } \\
\%\end{array}$ & $\begin{array}{c}\text { Caspase-3 activity, } \\
\text { OD value }\end{array}$ \\
\hline Blank control & $5.22 \pm 1.13$ & $0.15 \pm 0.02$ \\
Negative control & $5.42 \pm 1.44$ & $0.16 \pm 0.02$ \\
Liposome & $4.96 \pm 1.11$ & $0.16 \pm 0.03$ \\
Transfected & $18.76 \pm 2.79^{\mathrm{a}}$ & $0.48 \pm 0.05^{\mathrm{a}}$ \\
\hline
\end{tabular}

The transfected group was compared with the other groups, which served as controls. ${ }^{\text {a }}<0.05$. Data are presented as the mean \pm standard error of the mean. OD, optical density.

transfection for 48, 72 and $96 \mathrm{~h}$, cell proliferation was significantly lower in the $\beta$-catenin-siRNA-transfected group than in the other groups $(\mathrm{P}=0.007)$. The blank control, negative control and liposome groups exhibited no significant differences in cell proliferation $(\mathrm{P}=0.255)$. Thus, $\beta$-catenin-siRNA-mediated silencing of $\beta$-catenin gene expression was able to inhibit the proliferation of SW480 cells.

$\beta$-catenin-siRNA influences the apoptosis of SW480 cells. The results presented in Fig. 4 and Table I reveal that, upon silencing the $\beta$-catenin gene by $\beta$-catenin-siRNA, the apoptosis rate of SW480 cells was significantly higher in the $\beta$-catenin-siRNA-transfected group than in the blank control, negative control and liposome groups $(18.76 \pm 2.79 \%$ vs. $5.22 \pm 1.13,5.42 \pm 1.44$ and $4.96 \pm 1.11 \%$, respectively; $\mathrm{P}=0.004$ ). There was no significant difference in apoptosis among the blank control, negative control and liposome groups $(\mathrm{P}=0.166)$. Thus, $\beta$-catenin-siRNA transfection inhibited the apoptosis of SW480 cells.

$\beta$-catenin-siRNA promotes the activation of caspase- 3 in SW480 cells. The results in Table I indicate that, following transfection with $\beta$-catenin-siRNA for $48 \mathrm{~h}$, the levels of caspase-3 activity in the harvested SW480 cells were $0.15 \pm 0.02,0.16 \pm 0.02,0.16 \pm 0.03$ and $0.48 \pm 0.05$ OD value $(\mathrm{P}=0.005)$ in the blank control, negative control, liposome and $\beta$-catenin-siRNA-transfected groups, respectively. Thus, transfection with $\beta$-catenin-siRNA was able to increase caspase- 3 activity in SW480 cells.

Influence of $\beta$-catenin-siRNA on SW480 cell invasion and metastasis. The number of SW480 cells passing through the Matrigel membrane that was inhibited by transfection with $\beta$-catenin-siRNA indicated the invasion ability of SW480 cells in vitro. The results in Table II show that the in vitro invasiveness of SW480 cells transfected with $\beta$-catenin-siRNA for 24,48 and $72 \mathrm{~h}$, which were subsequently seeded into the invasion chamber and observed $24 \mathrm{~h}$ later, decreased following $\beta$-catenin-siRNA transfection $(\mathrm{P}=0.007)$, while no significant differences were observed among the different control groups $(\mathrm{P}=0.157)$. Following transfection with $\beta$-catenin-siRNA for 48 and $72 \mathrm{~h}$, the number of cells infiltrating the membrane in the $\beta$-catenin-siRNA-transfected group was significantly
Table II. Influence of small interference RNA targeting $\beta$-catenin on the invasive ability of SW480 cells in vitro.

Transfection, cell number

\begin{tabular}{llll}
\cline { 2 - 4 } Group & $24 \mathrm{~h}$ & $48 \mathrm{~h}$ & $72 \mathrm{~h}$ \\
\hline Blank control & $88 \pm 12$ & $89 \pm 10$ & $84 \pm 15$ \\
Negative control & $87 \pm 9$ & $85 \pm 13$ & $86 \pm 12$ \\
Liposome & $84 \pm 14$ & $82 \pm 11$ & $83 \pm 6$ \\
Transfected & $80 \pm 18$ & $51 \pm 10^{\mathrm{a}, \mathrm{b}}$ & $33 \pm 4^{\mathrm{a}-\mathrm{c}}$ \\
\hline
\end{tabular}

The transfected group was compared with the other groups, which served as controls. ${ }^{\mathrm{a}} \mathrm{P}<0.05$ vs. the same group at $24 \mathrm{~h},{ }^{\mathrm{b}} \mathrm{P}<0.05$ vs. the same group at $48 \mathrm{~h},{ }^{\mathrm{c}} \mathrm{P}<0.05 \mathrm{vs}$. the same group at $72 \mathrm{~h}$. Data are presented as the mean \pm standard error of the mean.
A

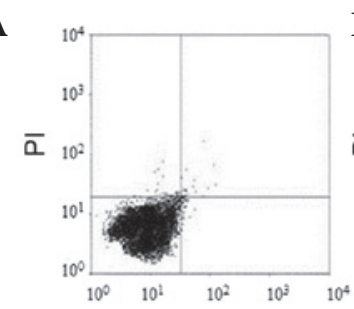

Annexin $\mathrm{V}$

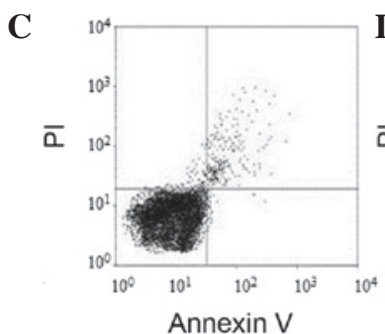

Annexin $\mathrm{V}$

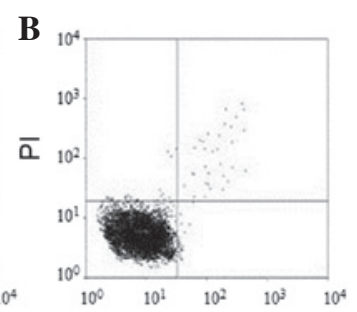

Annexin $\mathrm{V}$

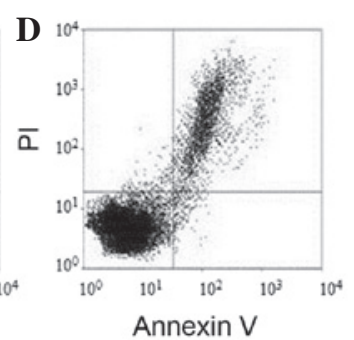

Figure 4. Apoptotic cells were detected by flow cytometry in the (A) blank control, (B) negative control, (C) liposome and (D) transfected groups. PI, propidium iodide.

reduced, compared with that in the other control groups $(\mathrm{P}=0.002)$, indicating that $\beta$-catenin-siRNA was able to reduce the invasiveness of SW480 cells in vitro in a time-dependent manner.

\section{Discussion}

Tumorigenesis, cancer progression and metastasis are complex processes (6). In recent years, the association between catenin and cancer has become well known and the number of studies on the association between cell adhesion molecules and malignant behavior has increased $(7,8)$. $\beta$-catenin is an important multifunctional cytoplasmic protein, which was initially considered as an intracellular molecule on the cell membrane that interacted with the calcium-dependent adhesion molecule E-cadherin (9). However, the isolation and cloning of $\beta$-catenin enabled to identify its dual function as mediator of cell adhesion and participant in Wnt signaling (10). $\beta$-catenin is located in the activation center of the Wnt signaling pathway, and is overexpressed in numerous tumors, including 
melanoma, leukemia, and small cell, breast and head and neck cancer (11-13). $\beta$-catenin is closely associated with various factors involved in the formation of tumors, and participates in multiple aspects of tumor development (14).

In the present study, siRNA technology was used, and double-stranded siRNA targeting $\beta$-catenin was synthesized (15-17). The experimental results indicated that specific siRNA targeting $\beta$-catenin could inhibit the expression of the $\beta$-catenin gene. RT-PCR and western blotting results demonstrated that, following transfection with $\beta$-catenin-siRNA, the mRNA and protein expression levels of $\beta$-catenin appeared to be significantly decreased, suggesting that siRNA could effectively block the Wnt signaling pathway.

The present study used RNA interference technology to explore the impact of silencing the $\beta$-catenin gene on the biological behavior of SW480 cells, and observed that the chemically synthesized $\beta$-catenin-siRNA could effectively inhibit the expression of $\beta$-catenin in SW480 cells. When the expression of the $\beta$-catenin gene was suppressed, the proliferation of SW480 cells was inhibited, and the apoptosis rate increased in these cells, which may be explained by the inhibition of apoptosis and promotion of proliferation mediated by $\beta$-catenin in SW480 cells. Thus, when $\beta$-catenin expression was inhibited, the apoptosis rate of SW480 cells increased. The anti-apoptotic mechanism of $\beta$-catenin is not yet entirely clear, but it may involve caspase-3, which is a major effector caspase in downstream apoptotic signal transduction pathways (18). In the present study, $\beta$-catenin-siRNA was used to inhibit $\beta$-catenin gene expression, which led to increased activity of caspase-3. Since $\beta$-catenin inhibits the apoptosis of SW480 cells and promotes their proliferation, the apoptosis of SW480 cells increases when the expression of $\beta$-catenin is disrupted. Studies on $\beta$-catenin and tumor invasiveness are limited. The present study has demonstrated that, upon silencing $\beta$-catenin gene expression, the number of SW480 cells penetrating the membrane in an invasion assay was significantly decreased, indicating that the invasive ability of these cells was significantly decreased. Thus, $\beta$-catenin-siRNA could effectively inhibit the expression of $\beta$-catenin in SW480 cells, and when $\beta$-catenin expression was suppressed, SW480 cell proliferation and invasion capacity were decreased, while apoptosis and caspase-3 activity were increased.

In the present study, SW480 cells with silenced $\beta$-catenin gene in the experimental group were characterized by slow proliferation in cell culture, low number of colonies formed in soft agar and markedly inhibited cell growth. Considering the experimental results obtained upon silencing the $\beta$-catenin gene and colon cancer cell proliferation, it is possible to speculate that in SW480 colon cancer cells specific siRNA targeting $\beta$-catenin may silence the $\beta$-catenin gene and sequentially silence its downstream genes. By silencing and inhibiting these upstream and downstream genes, the proliferation of SW480 cells was inhibited in vitro. Therefore, it can be proposed that, by interfering with the Wnt signaling pathway, $\beta$-catenin may act as a potential target gene for the treatment of colon cancer. However, further studies are required to guarantee that $\beta$-catenin-siRNA technology is stable, effective and secure to be used in clinical practice.

\section{Acknowledgements}

The present study was partly supported by grants from the Natural Science Foundation of Hubei Province (Wuhan, China; grant nos. 302-132139 and 302-131703).

\section{References}

1. Fodde R and Brabletz T: Wnt/beta-catenin signaling in cancer stemness and malignant behavior. Curr Opin Cell Biol 19: 150-158, 2007.

2. Ring A, Kim YM and Kahn M: Wnt/catenin signaling in adult stem cell physiology and disease. Stem Cell Rev 10: 512-525, 2014.

3. Fan K, Li N, Qi J, Yin P, Zhao C, Wang L, Li Z and Zha X: Wnt/ $\beta$-catenin signaling induces the transcription of cystathionine- $\gamma$-lyase, a stimulator of tumor in colon cancer. Cell Signal 26: 2801-2808, 2014.

4. Lazarova DL, Wong T, Chiaro C, Drago E and Bordonaro M: p300 influences butyrate-mediated WNT hyperactivation in colorectal cancer cells. J Cancer 4: 491-501, 2013.

5. Kumawat K, Koopmans $\mathrm{T}$ and Gosens $\mathrm{R}$ : $\beta$-catenin as a regulator and therapeutic target for asthmatic airway remodeling. Expert Opin Ther Targets 18: 1023-1034, 2014.

6. Denais $\mathrm{C}$ and Lammerding J: Nuclear mechanics in cancer. Adv Exp Med Biol 773: 435-470, 2014.

7. Tan CW, Gardiner BS, Hirokawa Y, Layton MJ, Smith DW and Burgess AW: Wnt signalling pathway parameters for mammalian cells. PLoS One 7: e31882, 2012.

8. Paul S and Dey A: Wnt signaling and cancer development: Therapeutic implication. Neoplasma 55: 165-176, 2008.

9. Lai T, Su C, Kuo W, Yeh Y, Kuo W, Tsai F, Tsai C, Weng Y and Huang $C$ : $\beta$-catenin plays a key role in metastasis of human hepatocellular carcinoma. Oncology Reports 26: 415-422, 2011.

10. Voronkov A and Krauss S: Wnt/beta-catenin signaling and small molecule inhibitors. Curr Pharm Des 19: 634-664, 2013.

11. Clevers $H$ and Nusse $R:$ Wnt/ $\beta$-catenin signaling and disease. Cell 149: 1192-1205, 2012.

12. Schmeel LC, Schmeel FC, Kim Y, Endo T, Lu D and Schmidt-Wolf IG: Targeting the Wnt/beta-catenin pathway in multiple myeloma. Anticancer Res 33: 4719-4726, 2013.

13. Yao H, Ashihara E and Maekawa T: Targeting the Wnt/ $\beta$-catenin signaling pathway in human cancers. Expert Opin Ther Targets 15: 873-887, 2011.

14. Behrens J: Control of beta-catenin signaling in tumor development. Ann N Y Acad Sci 910: 21-35, 2000.

15. Flemr M, Malik R, Franke V, Nejepinska J, Sedlacek R, Vlahovicek K and Svoboda P: A retrotransposon-driven dicer isoform directs endogenous small interfering RNA production in mouse oocytes. Cell 155: 807-816, 2013.

16. Dogini DB, Pascoal VD, Avansini SH, Vieira AS, Pereira TC and Lopes-Cendes I: The new world of RNAs. Genet Mol Biol 37 (Suppl 1): S285-S293, 2014.

17. Fellmann $C$ and Lowe SW: Stable RNA interference rules for silencing. Nat Cell Biol 16: 10-18, 2014.

18. Ismail B, Ghezali L, Gueye R, Limami Y, Pouget C, Leger DY, Martin F, Beneytout J, Duroux J, Diab-Assaf M, et al: Novel methylsulfonyl chalcones as potential antiproliferative drugs for human prostate cancer: Involvement of the intrinsic pathway of apoptosis. Int J Oncol 43: 1160-1168, 2013. 